\title{
Short Rayleigh length free electron laser: Experiments and simulations
}

\author{
P. P. Crooker, W. B. Colson, J. Blau, D. Burggraff, and J. Sans Aguilar \\ Physics Department, Naval Postgraduate School, Monterey, California 93943, USA \\ S. Benson, G. Neil, M. Shinn, and P. Evtushenko \\ Thomas Jefferson National Accelerator Laboratory, Newport News, Virginia 23606, USA
}

(Received 31 January 2008; published 17 September 2008)

\begin{abstract}
We report experiments at Jefferson National Accelerator Facility (Jlab) and computer simulations performed at the Naval Postgraduate School (NPS) designed to probe the small Rayleigh length regime. We compare the gain, power, and sensitivity to mirror and electron beam misalignments as a function of decreasing Rayleigh length. The agreement is quite good, with experiments and simulations showing comparable trends as the Rayleigh length is decreased. In particular, we find that the gain and power $d o$ not decrease substantially at short Rayleigh length, contrary to a common Gaussian-mode filling factor argument. Within currently achievable alignment tolerances, the gain and power are still acceptable for FEL operation.
\end{abstract}

DOI: 10.1103/PhysRevSTAB.11.090701

PACS numbers: 41.60.Cr

\section{INTRODUCTION}

Early theoretical models [1-3] of the single-pass gain of a free electron laser (FEL) took into account the overlap between the electron beam and the lowest order optical cavity mode. The overlap is described by a filling factor, which is just the area of the electron beam divided by the optical mode area. The gain $G$ is defined as the fractional optical power increase $\Delta P / P$ in one pass through the FEL undulator in the weak-field regime, not including losses due to end effects at the mirrors. The optical mode is characterized by Rayleigh length $Z_{0}$, which is the distance from the waist over which the beam area of the fundamental Gaussian-mode doubles (determined by the cavity length and the radius of curvature of the end mirrors). A simplistic and conventional approach to estimating the gain assumes a Gaussian fundamental optical mode and averages the filling factor over the length $L$ of the undulator [3]. The result is

$$
G=\frac{G_{0} / \sqrt{3}}{Z_{0} / L+L /\left(12 Z_{0}\right)},
$$

where $G_{0}$ is the peak gain in (1) at $Z_{0}=L / \sqrt{12}$. From this expression it can be seen that the gain falls off rapidly at small $Z_{0}$. However, it should be noted that this simple theory does not take into account the self-consistent interaction between the electrons and the diffracting optical field as they evolve along the undulator length. At small $Z_{0}$, the optical mode becomes distorted-it no longer matches the cavity fundamental mode-so we should expect Eq. (1) to fail in this regime.

Computer simulations at the Naval Postgraduate School (NPS) $[4,5]$ have shown that this simple theory does not describe the actual behavior of FEL gain. Figure 1 shows the simulated gain $G / G_{0}$ versus Rayleigh length (normal- ized by the undulator length: $z_{0}=Z_{0} / L$ ) as the FEL peak gain is increased. Clearly the simple theory holds only when the peak gain is less than a few percent.

For a cold cavity (no gain), a characteristic feature of short Rayleigh length resonators is their extreme sensitivity to mirror misalignments [6]. In particular, a small tilt or shift of a mirror can cause a large tilt of the optical mode axis. It is therefore important to know how mirror tilt and shift affects a laser with gain, such as ours. Note also that, since the time scale of mirror vibrations (milliseconds) is orders of magnitude greater than the round-trip time of photons in the resonator (microseconds), a study of static mirror misalignments will allow us to see how dynamic misalignments-i.e., mirror vibrations-will affect laser stability.

The motivation for this paper is therefore to compare the NPS simulations with short Rayleigh length experiments

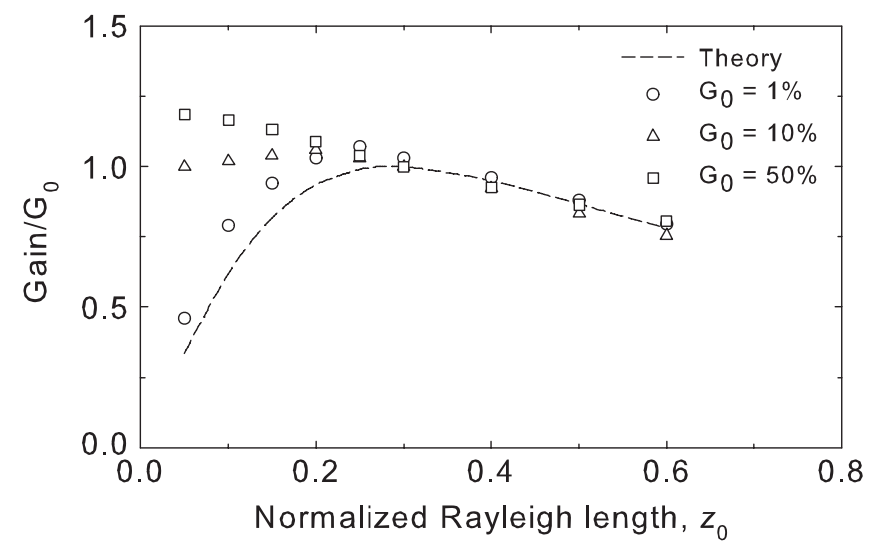

FIG. 1. Simulations of weak-field gain versus normalized $z_{0}$ for increasing values of $G_{0}$. The dashed curve is the gain predicted by Eq. (1). 
performed at the Thomas Jefferson National Accelerator Laboratory (JLab) FEL [7]. Simulations and experiments determine both the gain and power as a function of Rayleigh length and show how the gain is affected by mirror tilt and shift and by electron beam tilt and shift.

\section{PARAMETERS}

Our parameter set is based on JLab operating conditions in August of 2006, using their $5.5 \mathrm{~cm}$ period, permanent magnet undulator. The electron beam energy is $115 \mathrm{MeV}$, the bunch charge is $108 \mathrm{pC}$, the FWHM pulse duration is $0.4 \mathrm{ps}$, and the rms electron beam radius is $0.2 \mathrm{~mm}$. The normalized transverse emittance is $7.5 \mathrm{~mm}$-mrad, and the longitudinal emittance is $70 \mathrm{keV}$-ps. The electron beam waist was approximately in the center of the undulator with an experimental $\beta$ function $\approx 80 \mathrm{~cm}$. The undulator period is $\lambda_{0}=5.5 \mathrm{~cm}$ with 30 periods, for a total length $L=1.65 \mathrm{~m}$. The $\mathrm{rms}$ undulator parameter $K=$ $e B_{\mathrm{rms}} \lambda_{0} / 2 \pi m c=0.81$, where $B_{\mathrm{rms}}$ is the rms undulator magnetic field, $e$ and $m$ are the electron charge and mass, and $c$ is the velocity of light [3]. The optical wavelength $\lambda=0.95 \mu \mathrm{m}$. The optical cavity length $S=32 \mathrm{~m}$ with a nominal value of $Z_{0}=70 \mathrm{~cm}$, and the output coupling is $5.9 \%$, corresponding to cavity quality factor $Q_{n}=17$. The undulator is centered in the optical cavity but the optical waist is slightly downstream of center and moves toward the center as $Z_{0}$ decreases.

To keep the results general, nondimensional units are used as follows: longitudinal lengths are scaled by the undulator length $L$ (i.e., $z_{0}=Z_{0} / L$ ), transverse lengths are scaled by $\sqrt{\lambda L / \pi}=0.706 \mathrm{~mm}$, and angles relative to the mirror axis are scaled by $\sqrt{\lambda / \pi L}=0.428 \mathrm{mrad}$.

\section{EXPERIMENTAL AND COMPUTATIONAL METHODS}

\section{A. Experimental methods}

For a cold cavity (no gain) with mirrors having identical radii of curvature $R$ and separation $S$, the normalized Rayleigh length $z_{0}$ of the optical mode is determined by

$$
z_{0}=\frac{Z_{0}}{L}=\sqrt{\frac{S(2 R-S)}{4 L^{2}}} .
$$

In an operating FEL, $R$ may change due to mirror heating, thereby changing $z_{0}$ and hence the gain $G$ and output optical power $P$. However, all our results were obtained at low power to avoid heating.

The Rayleigh length of the optical mode is varied by adjusting the radius of curvature $R$ of a cavity mirror. The output coupling mirror is sapphire with a $\mathrm{ZnSe} / \mathrm{ThF}_{4}$ dielectric coating and fixed $R=16.0 \mathrm{~m}$. The highreflecting mirror has a coated silicon substrate glued to a heater-controlled deformable mirror assembly. This mirror had curvature radius $R=16.3 \mathrm{~m}$ with the heater off; with the heater on, $R$ decreased linearly with the heater power.

The Rayleigh length was calibrated two ways. First, one can calculate it from the measured mirror radius of curvature $R$. Since $R$ is a known function of heater power, one can show that a change in $R$ causes a corresponding change in $z_{0}$ according to

$$
\frac{d z_{0}}{z_{0}}=\frac{M}{2} \frac{d R}{R} .
$$

Here the magnification $M$, the ratio of the mode area at the mirror to that at the waist, is given by $M=1+\left(S / 2 L z_{0}\right)^{2}$. When the magnification is large $\left(M \sim 10^{4}\right)$, a small inaccuracy in $d R / R$ leads to a large inaccuracy in $d z_{0} / z_{0}$. It should be noted that (2) and (3) above, as well as (4) below, apply to symmetrical resonators. However, since only one mirror was deformed, the optical waist center was slightly downstream of the undulator center for long Rayleigh length, moving toward the center for short Rayleigh length. Although this asymmetry was also included in the simulations, it was not found to be a large effect compared to other changes in the optical beam. In fact, exact equations were used for all calculations but since asymmetric effects were negligible, both in the experiment and in the simulations, only the symmetric equations have been included here.

The second method for finding $z_{0}$ requires measuring the mode radius $w_{m}$ on the mirror directly. Using the wavelength $\lambda$ and dimensional values of $S$ and $w_{m}$, one can show that

$$
z_{0}=\frac{\pi w_{m}^{2}}{2 \lambda L}\left[1-\sqrt{1-\left(\frac{\lambda S}{\pi w_{m}^{2}}\right)^{2}}\right] \approx \frac{\lambda S^{2}}{4 \pi w_{m}^{2} L} .
$$

In this case the accuracy is much improved since the magnification does not enter the equation.

For small magnification, the first method is the most accurate; for large magnification, the second method is more accurate. In the second method, gain will cause mode distortion which alters the calibration. To minimize this effect, the smallest gain possible was used during these calibration measurements. However as a check, where there was overlap both methods were in good agreement.

\section{B. Computational methods}

The wavefront propagation technique used in the simulations has been described in detail elsewhere $[2,5,8]$. At each time step, it uses the relativistic Lorentz force equations to determine the electron motion in the presence of the undulator and optical fields, and the parabolic wave equation to evolve the optical wavefront in transverse directions $x$ and $y$ and time $t$. The simulation can follow multiple and arbitrary transverse optical modes as they interact with the electron beam and bounce back and forth in the optical cavity. Mirror transmission and edge losses are included. For determining gain $G$, we keep the simu- 
lation in weak optical fields, allowing it to evolve through the required number of undulator passes until the gain is constant.

Recent improvements to our program include the ability to tilt or shift either an end mirror or the electron beam. We have also incorporated a variable coordinate system which expands with the rapidly expanding optical mode as it proceeds through the system [5].

\section{GAIN VERSUS RAYLEIGH LENGTH}

The experimental gain was found by fitting the output power to a growing exponential. The range of the fit was chosen to keep the power less than $5 \%$ of the saturated power. The falloff after the electron beam was turned off was fit to a decaying exponential to determine the resonator losses.

The effect of normalized $z_{0}$ on the gain is shown in Fig. 2. The squares show JLab experimental data; the connected circles show simulations using the same input parameters. The dashed line is the simple Gaussian theory of Eq. (1). The simulations and experiment agree well with each other.

The most dramatic result of Fig. 2 is that neither the simulation nor the experiment show the downward trend of the simple theory at small $z_{0}$ (dashed line) as mentioned in Sec. I. While the simple theory uses only the fundamental Gaussian optical mode for all $z_{0}$, both the experiments and simulations show that, for small $z_{0}$, the mode is not the fundamental. Figure 3 shows the simulated optical mode along the undulator for $z_{0}=0.05$ in the small signal regime. The theoretical optical mode profile (gray line) is calculated from the simple theory using $z_{0}$ derived from Eq. (2). The actual mode profile (black line) is narrower, a result of selective optical gain along the narrow electron beam. Because the optical mode is narrower, the 1/e mode

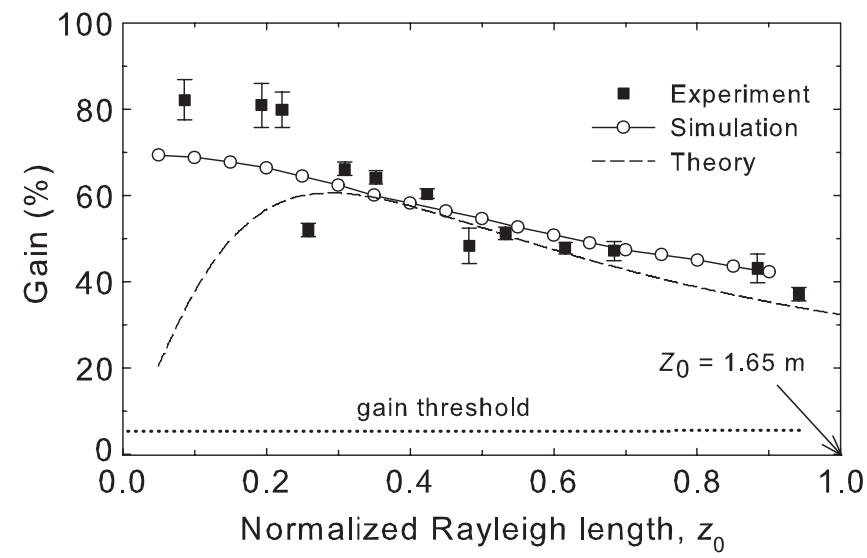

FIG. 2. Dependence of gain on normalized Rayleigh length $z_{0}$. The dashed line is derived from Eq. (1). The dotted line is the cavity loss due to output coupling (5.9\%); the gain must at least overcome this loss. The dimensional Rayleigh length at $z_{0}=1$ is $Z_{0}=1.65 \mathrm{~m}$.

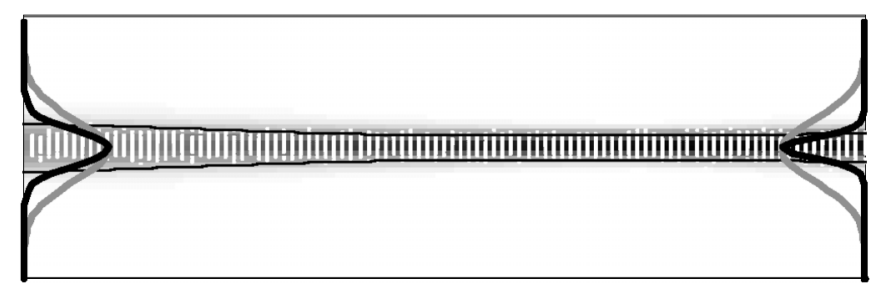

FIG. 3. Profile of the optical mode (gray shading) and electron beam (vertical white lines at each calculation step) along the undulator for $z_{0}=0.05$ in the small signal regime. The gray optical mode profile uses $z_{0}$ from Eq. (2) and the simple theory; the black profile is the actual mode profile from the simulation. The narrowness of the simulation profile is due to selective amplification along the narrow electron beam.

radius at the cavity mirrors is about $40 \%$ of the mirror radius. We have demonstrated the lack of mirror edge effects by enlarging the mirrors in the simulations with no change in the results.

For larger $z_{0}\left(z_{0}>0.3\right)$, the theory, simulation, and experiment are in agreement, with the weak-field gain varying as $1 / z_{0}$.

\section{POWER}

Figure 4 shows both the experimental and simulated power in strong optical fields, at saturation, as a function of $z_{0}$. Except for $z_{0}<0.3$ where the experimental optical beam edges were cut off by the optical transport system to the detector, the $z_{0}$ dependence of the two results is in good agreement. Similar agreement has been reported for previous NPS simulations of JLab experiments [9]. Note also that, since the gain remains above threshold, there is no tendency for the power to decrease substantially at low $z_{0}$ for either the simulation or the experiment.

\section{MIRROR TILT}

Tilting of the cavity mirrors may be caused by system vibrations or uneven heating of the mirror supports. Tilting a mirror causes its center of curvature to be offset from the

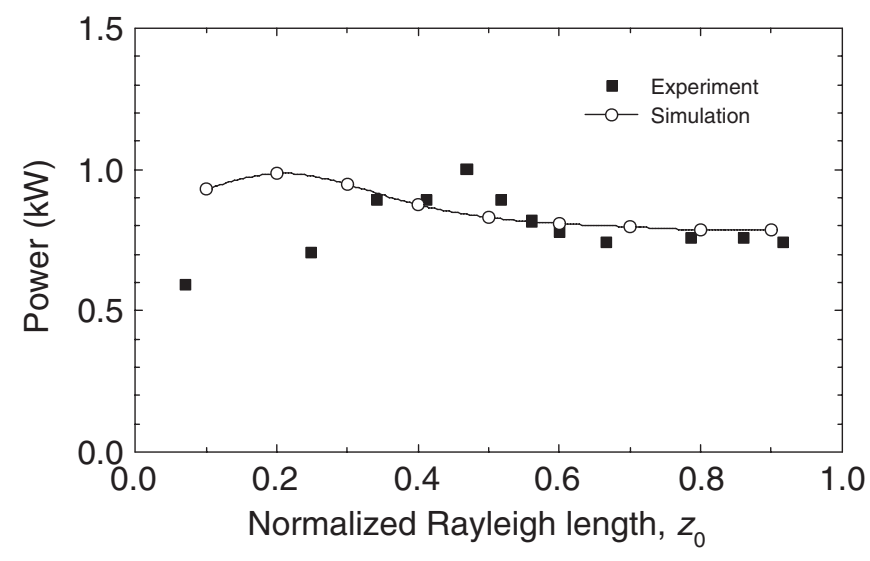

FIG. 4. Dependence of output power in a macropulse on $z_{0}$. 


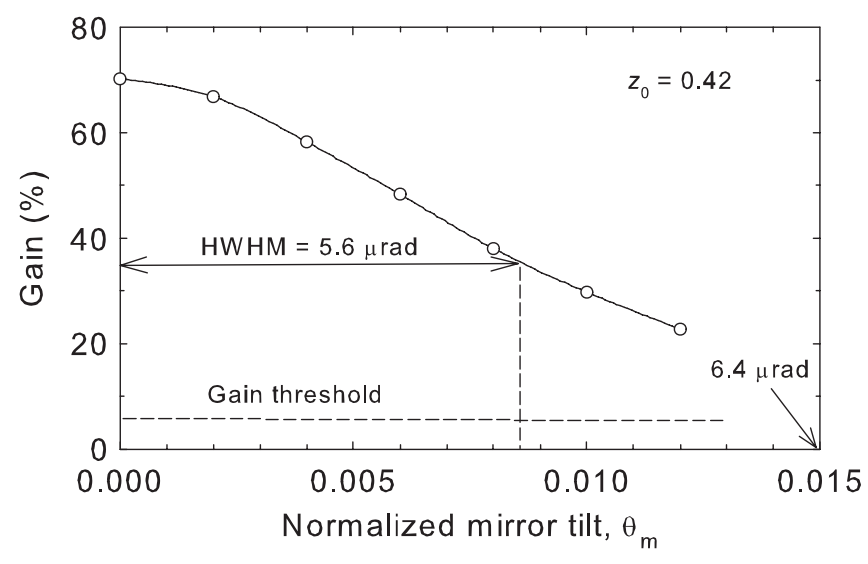

FIG. 5. Simulations of gain vs normalized mirror tilt angle for $z_{0}=0.42$. The angle at half-width half-maximum (HWHM) gain and the maximum angle in $\mu \mathrm{rad}$ are shown.

original cavity axis and redefines a new cavity axis which is no longer aligned with the electron beam. As the mirror is tilted further the electron beam/optical mode overlap is reduced with an accompanying falloff of the gain.

Figure 5 shows the simulated gain as the output mirror tilt angle is increased when $z_{0}=0.42$. The gain decreases monotonically as the tilt increases; at the half-width halfmaximum (HWHM) point the normalized tilt is $\theta_{m}=$ 0.0087 , corresponding to an actual tilt of $5.6 \mu \mathrm{rad}$.

Rather than measure complete gain curves as in Fig. 5, the experimentally determined HWHM angle was determined by simply tilting the mirror until the gain was reduced to half its maximum value. Figure 6 shows the HWHM values versus $z_{0}$ for both experiment and simulations. The agreement is good over the measured range of $z_{0}$. Note the general upward trend with increasing $z_{0}$ for both simulation and experiment: this trend is because the cavity is less sensitive to mirror tilt at larger $z_{0}$.

\section{MIRROR SHIFT}

In addition to tilt, vibrations may also cause the mirrors to shift transversely. As in the case of mirror tilt, transverse

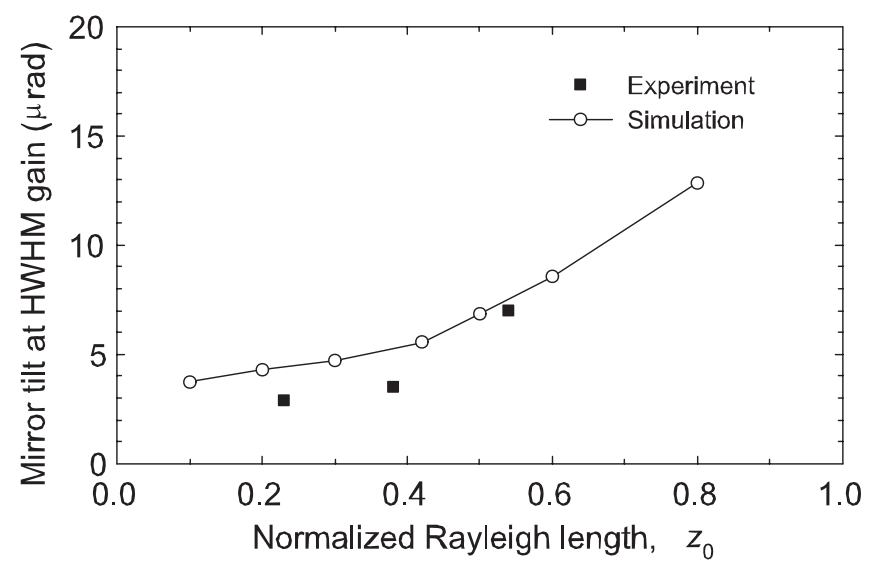

FIG. 6. Mirror tilt at HWHM gain versus $z_{0}$.

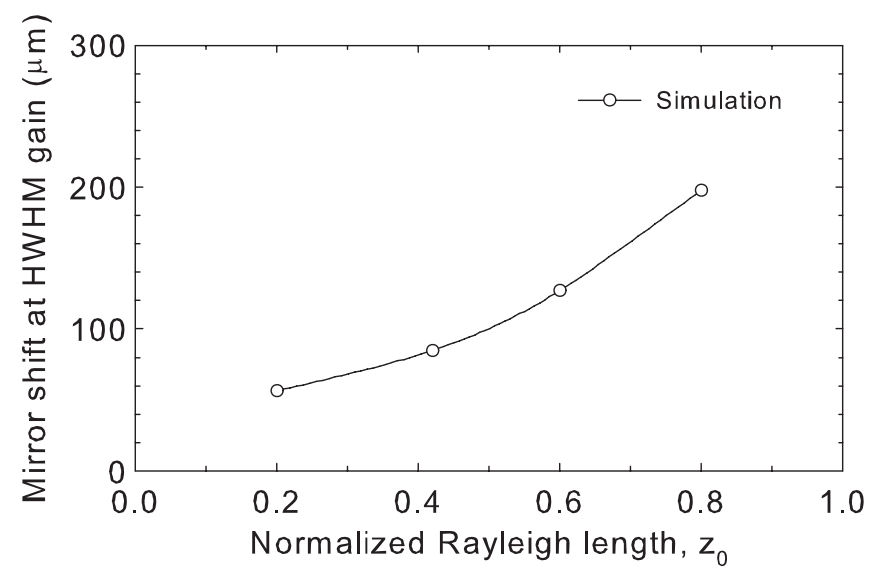

FIG. 7. Mirror shift at HWHM gain versus $z_{0}$.

mirror shift also displaces the mirror center of curvature and effectively tilts the cavity axis, thereby reducing the optical mode/electron beam overlap and decreasing the gain. Plots of gain versus mirror shift (not shown) are qualitatively similar to Fig. 5; again we use the mirror shift at HWHM gain as a measure of allowable mirror shift.

Figure 7 shows simulations of the HWHM mirror shifts versus $z_{0}$. No experimental data corresponding to these simulations were taken. The monotonic increase of allowable mirror shift with increasing $z_{0}$ is quite similar to the mirror tilt behavior shown in Fig. 5. As $z_{0}$ is increased, a fixed mirror shift causes less cavity axis tilt, thereby allowing larger mirror shifts before the gain is significantly reduced.

\section{ELECTRON BEAM TILT}

The position of the electron beam in the undulator is determined by steering magnets. Vibration of the magnets or fluctuations in the magnetic field can cause the beam to tilt and/or undergo a transverse shift in the undulator. Experiments were performed by first tilting the beam and then shifting the beam transversely until the gain was maximized. The simulations emulated this procedure by first tilting the beam about the undulator center and then maximizing the gain with transverse shift. However, the amount of shift required to maximize the gain was negligible. The results are shown in Fig. 8, where the electron beam has been tilted in the plane of the undulator field. At large $z_{0}$, experiment and simulations are in agreement. For small $z_{0}$, the simulation flattens out as a result of mirror edge effects; nevertheless, the simulations still show a larger allowed tilt than is seen by the experiments.

One possible explanation for the experimental behavior is a nonlinearity in the electron beam transport for the experimental setup. The offset in the upstream focusing lenses for a given angular offset in the undulator was very large. For example, a $1.5 \mathrm{mrad}$ beam tilt in the undulator required a $2 \mathrm{~cm}$ offset in the upstream quadrupoles. Such a 


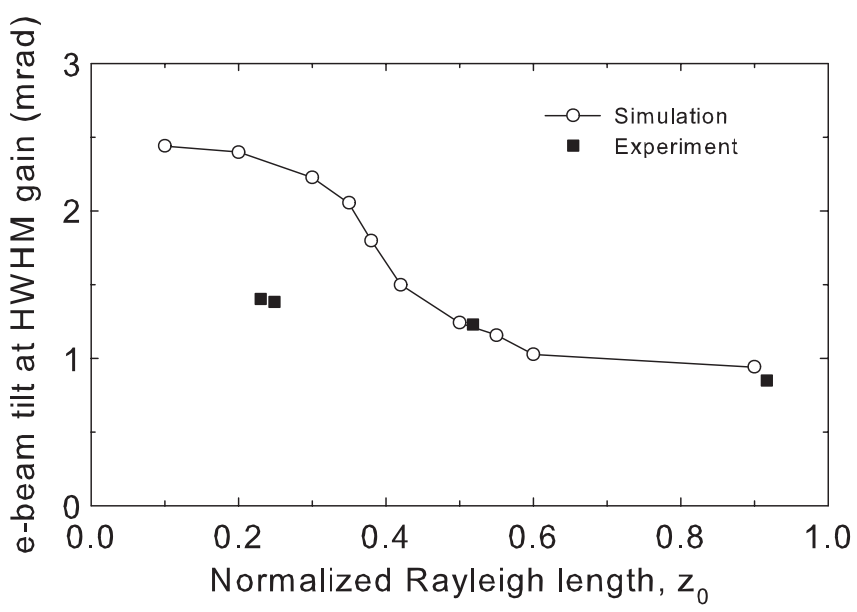

FIG. 8. Electron beam tilt at HWHM gain versus $z_{0}$.

large offset can lead to nonlinear behavior that can dilute the longitudinal phase space, leading to a reduced peak current. This hypothesis will be tested in the future by using a different set of steering coils to reduce the beam offset in the upstream focusing elements for a given angular offset in the undulator.

Note that both experiment and simulation show a general increase in allowed e-beam tilt as $z_{0}$ decreases. At lower $z_{0}$, the mode divergence is larger and hence larger electron beam tilts can be accommodated without losing the electron beam/optical mode overlap.

\section{ELECTRON BEAM SHIFT}

A shift in the electron beam position without any tilt can be caused by errors in magnet steering or vibration of the optical cavity about the existing electron beam. When the shift becomes larger than the optical mode envelope, we expect the gain to decrease. Figure 9 shows simulation results where the beam is shifted in the plane of the undulator magnetic field. The agreement with the single experimental point is good.

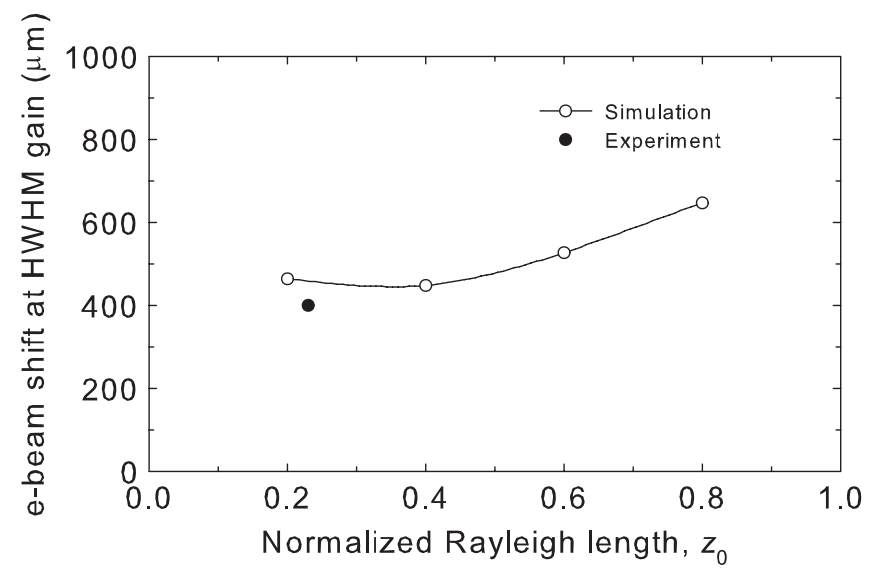

FIG. 9. Electron beam shift at HWHM gain versus $z_{0}$.
Note that here the allowed beam shift increases with increasing $z_{0}$, whereas the allowed beam tilt decreases with increasing $z_{0}$. As $z_{0}$ increases, the mode waist also increases so that greater beam shifts can be tolerated without reducing the electron beam/optical mode overlap in the center of the undulator.

\section{CONCLUSIONS}

We have compared the results of short Rayleigh length FEL experiments at JLab with computer simulations performed at NPS. In particular, both the experiments and the simulations demonstrate that the gain of the FEL does not decrease with decreasing $z_{0}$. This is due to the optical mode adjusting its shape to conform to the electron beam, rather than remaining in the fundamental mode (determined by the cavity length and mirror radius of curvature) as the simple theory assumes. It is only for very small gains of $\sim 1 \%$ that the optical cavity geometry determines the optical mode.

We also find that the FEL is quite stable at small $z_{0}$. As the Rayleigh length tends to zero, JLab experiments and NPS simulations of mirror and electron beam misalignments remain in good agreement. Within currently achievable alignment tolerances, the gain and power are still acceptable for FEL operation.

\section{ACKNOWLEDGMENTS}

Support for this project was provided by the High Energy Laser Joint Technology Office and the Office of Naval Research.

[1] J. M. J. Madey, J. Appl. Phys. 42, 1906 (1971).

[2] W. B. Colson, in Laser Handbook, edited by W. B. Colson, C. Pelligrini, and A. Renieri (North-Holland, Amsterdam, 1990), Vol. 6, Chap. 5, Sec. 18.

[3] W. B. Colson and P. Elleaume, Appl. Phys. B 29, 101 (1982).

[4] D. Small, R. K. Wong, W. B. Colson, and R. L. Armstead, Nucl. Instrum. Methods Phys. Res., Sect. A 393, 262 (1997).

[5] W. B. Colson, J. Blau, R. L. Armstead, P. P. Crooker, R. Vigil, T. Voughs, and B.W. Williams, Phys. Rev. ST Accel. Beams 9, 030703 (2006).

[6] A.E. Siegman, Lasers (University Science Books, Sausalito, 1986), Secs. 15.4 and 19.4.

[7] S. Benson et al., Proceedings of the 2007 Particle Accelerator Conference, Albuquerque, New Mexico, 2007 (IEEE, Albuquerque, New Mexico, 2007), http://accelconf.web.cern.ch/AccelConf/p07/papers/ MMOOAAB03.pdf.

[8] J. Blau, Ph.D. thesis, Naval Postgraduate School, 2002.

[9] R. D. McGinnis, J. Blau, W. B. Colson, D. Massey, P. P. Crooker, A. Christodolou, and D. Lampiris, Nucl. Instrum. Methods Phys. Res., Sect. A 475, 178 (2001). 